\title{
Editorial
}

\section{Low Dialysate Calcium: Between Low Arterial Calcification and Improved Low Bone Turnover}

\author{
Mario Cozzolino $^{a}$ Stefano Carugo $^{b}$ \\ ${ }^{a}$ Renal Division and Laboratory of Experimental Nephrology, and ${ }^{b}$ Cardiology Unit, Department of Health Sciences, \\ San Paolo Hospital, University of Milan, Milan, Italy
}

Vascular calcification and cardiovascular diseases pose a major problem in advanced chronic kidney disease (CKD) patients, constituting a major cause of morbidity and mortality in dialysis population. CKD patients, beyond classic risk factors, also retain 'non-classic' cardiovascular risk factors such as dialysis vintage, anaemia, hyperhomocysteinaemia, endothelial dysfunction, chronic microinflammation, and mineral metabolism alterations [1].

Exposure to elevated calcium concentrations can influence the low-turnover bone disease and vascular calcification in CKD. The present issue of the Journal, Lu et al. [2] investigates the efficacy and safety of low calcium dialysate in hemodialysis patients with serum iPTH lower than $100 \mathrm{pg} / \mathrm{ml}$. Interestingly, calcium concentration of $1.25 \mathrm{mmol} / \mathrm{l}$ is found to be associated with improved bone mineral parameters and cardiovascular calcification indexes, such as carotid intima-media thickness and carotid artery and abdominal aorta calcification scores. These data are originating 40 years since the publication by Fournier et al. [3], in which these authors have observed that plasma concentration of iPTH was lowest with the combination of high dialysate calcium and low plasma phosphate and highest with the combination of low dialysate calcium and high plasma phosphate. They con-

\section{KARGER}

E-Mail karger@karger.com

www.karger.com/bpu cluded that both dialysate calcium and plasma phosphate were important determinants of the parathyroid function in HD patients.

During the last decade, several studies have demonstrated that this conclusion could be a subject for discussion. Recently, in a randomized clinical trial, Ok et al. [4] showed that lowering dialysate calcium levels slowed the progression of coronary artery calcification and improved the bone turnover in patients on hemodialysis with baseline iPTH $\leq 300 \mathrm{pg} / \mathrm{ml}$. In addition, Gonzalez-Parra et al. [5] described that the individualization of dialysate calcium concentration according to baseline pre-dialysis serum calcium may prevent major excursions in post-dialysis serum calcium and iPTH levels.

The dialysate calcium concentration for HD patients can be adjusted to manage the calcium-phosphate balance, to ameliorate bone metabolism, and to reduce accelerated vascular calcification. The more appropriate dialysate calcium concentration should be prescribed to each individual patient depending on several factors relating to the calcium load. A lower dialysate calcium concentration of $1.25 \mathrm{mmol} / \mathrm{l}$ may increase the use of vitamin $\mathrm{D}$ in clinical practice, with reduced risk of extraskeletal calcifications. Low calcium baths may turn out to be useful in the setting of adynamic bone disease,
(C) 2016 S. Karger AG Basel

0253-5068/16/0421-0001\$39.50/0
Mario Cozzolino, MD, PhD, FERA

Renal Division and Laboratory of Experimental Nephrology Dipartimento di Scienze della Salute, Università di Milano, Renal Division San Paolo Hospital, Via Antonio di Rudinì, 8, IT-20142 Milan (Italy) E-Mail mario.cozzolino@unimi.it 
where an increase in bone turnover is desirable. However, low calcium levels in the dialysate may also induce cardiac arrhythmias during dialysis sessions, with intradialytic hypotension. Nowadays, determining the ideal dialysate calcium seems to be a difficult task because of the complex pathophysiology of bone and mineral metabolism in CKD patients. To optimize management of the abnormal calcium balance, other aspects of this dis- order need to be more fully clarified, that is aspects such as treatment of hyperphosphatemia and secondary hyperparathyroidism, as well as the emergence of a multitude of different hemodialysis techniques need to be ascertained completely. In conclusion, we need to maintain flexibility with dialysis, and calcium dialysate should be individualized to meet the specific requirements of CKD patients.

\section{References}

1 Cozzolino M, Ureña-Torres $P$, Vervloet MG, Brandenburg V, Bover J, Goldsmith D, Larsson TE, Massy ZA, Mazzaferro S; CKD-MBD Working Group of ERA-EDTA: Is chronic kidney disease-mineral bone disorder (CKDMBD) really a syndrome? Nephrol Dial Transplant 2014;29:1815-1820.

2 Lu J, Yi Y, Xiong Z, Cheng X, Hu J, Hang H, Cheng J, Peng W: The study of low calcium dialysate on elderly hemodialysis patients with secondary hypoparathyroidism. Blood Purif 2016;42:3-8.
3 Fournier AE, Arnaud CD, Johnson WJ, Taylor WF, Goldsmith RS: Etiology of hyperparathyroidism and bone disease during chronic hemodialysis. II. Factors affecting serum immunoreactive parathyroid hormone. J Clin Invest 1971;50:599-605.

4 Ok E, Asci G, Bayraktaroglu S, Toz H, Ozkahya M, Yilmaz M, Kircelli F, Sevinc Ok E, Ceylan N, Duman S, Cirit M, MonierFaugere MC, Malluche HH: Reduction of dialysate calcium level reduces progression of coronary artery calcification and improves low bone turnover in patients on hemodialysis. J Am Soc Nephrol 2015; pii: ASN.2015030268.
5 Gonzalez-Parra E, Gonzalez-Casaus ML, Arenas MD, Sainz-Prestel V, Gonzalez-Espinoza L, Muñoz-Rodriguez MA, Tabikh A, Egido J, Ortiz A: Individualization of dialysate calcium concentration according to baseline pre-dialysis serum calcium. Blood Purif 2014; 38:224-233. 